\title{
Chemical Quantification of Atomic-scale EDS Maps under Thin Specimen Conditions
}

Ping $\mathrm{Lu}^{1}$, Jie Xiong ${ }^{2}$, Mark Van Benthem ${ }^{1}$, and Quanxi Jia ${ }^{2}$

${ }^{1}$ Sandia National Laboratories, PO Box 5800, MS 1411, Albuquerque, NM 87185-1411 USA $^{2}$ Center for Integrated Nanotechnologies, Los Alamos national laboratory, Los Alamos, NM 87545, USA

Recent technical advances in scanning transmission electron microscopy (STEM) and xray detector technology have made it possible to do atomic-resolution chemical mapping using energy-dispersive x-ray spectroscopy (EDS). Routine atomic-resolution microanalysis based on STEM-EDS has been demonstrated using a microscope, based on a FEI Titan ${ }^{\mathrm{TM}}$ platform, which combines a high-brightness X-FEG electron source, probe spherical aberration correction technology and quadruple windowless silicon drift EDS detectors[1-5]. However, attempts to quantify chemical compositions at atomic scale have been very limited so far [5].

In this work, we report our effort to quantify atomic-scale chemical maps obtained by STEM-EDS spectral imaging. Direct quantification of chemical composition at the atomic columns, in general, is very complicated due to multiple effects, including electron beam spreading and channeling as well as de-channeling. Furthermore, these effects are dependent on concentration at the atomic columns [1,6-8]. Effects of beam spread and de-channeling can lead to relative contributions from neighboring columns, or so-called "cross-talk", even when a fine probe is focused on a specific column of atoms. Figure 1 schematically shows x-ray generation in an incoherent mode in STEM. The EDS signal resulted from a particular section of specimen at thickness ( $\mathrm{z}$ ) is obtained by direct convolution of the electron wave function at that thickness with effective EDS ionization potential localized at the atomic column. Integrating the contribution over specimen thickness gives rise to the total signal from the specimen. Under thin specimen conditions such that the effects of de-channeling and beam spread are small, the EDS signal can be localized to atomic columns; provided that the effective EDS potential is also localized. Under such conditions, EDS x-ray counts resulting from individual atomic columns can be calculated by fitting Gaussian peaks at each of atomic column position.

An example that illustrates the method is shown in Figure 2. The EDS maps in Fig. 2 are extracted from a STEM-EDS spectral imaging dataset for a thin $\mathrm{SrTiO}_{3}$ bulk sample in [100] zone axis. The x-ray line profiles (Fig. 2c) were obtained by integrating EDS maps along the vertical direction. The between-column intensities (valleys of line profiles) are from contributions of neighboring columns or overlap of neighboring Gaussian peaks. Gaussian peak fitting of the curve was used to extract the contribution from each atomic column in Fig. $2 \mathrm{c}$. We determined full widths at half maximum (FWHM) of the Gaussian peaks to be $0.28 \mathrm{~nm}$ and 0.26 $\mathrm{nm}$ for $\mathrm{Sr}$ and Ti profiles, respectively. The x-ray counts under each Gaussian peak at the atomic column can be used for further quantitative chemical quantification. Using this method, we have quantified chemical composition at the atomic-scale across a ferromagnetic $\mathrm{La}_{0.7} \mathrm{Sr}_{0.3} \mathrm{MnO}_{3}$ and antiferromagnetic $\mathrm{BiFeO}_{3}$ interface $[9,10]$.

\section{References:}

[1] A.J. D’Alfonso, B. Freitag, D.Klenov, and L.J. Allen, Phys. Rev. B 81, 10010 (2010). 
[2] M.W. Chu, S.C. Liou, C.P.Chang, F.S. Choa, and C.H.Chen, Phys, Rev. Lett. 104, 196101 (2010)

[3] Dmitri O. Klenov and Joshua M.O. Zide, App. Phys. Lett. 99, 141904 (2011);

[4] H.S. von Harrach, P.Dona, B. Freitag, H. Soltau, A. Niculae, and M. Rohde, Microsc. Microanal. 15 (suppl.2) 208, (2009)

[5] P. Kotula, D. O. Klenov, and H.S. Von Harrach, Microsc. Microanal 18, 691 (2012).

[6] Muller, D.A., Fitting Kourkoutis, L., Murfitt, M., Song, J. H., Silcox, J., Dellby, N., and Krivanek, O.L., Science 319, 1073-1076 (2008)

[7] P. Wang, A.J. D’Alfonso, S.D. Findlay, L. J. Allen, and A. L. Bleloch, Phys. Rev. Lett. 101, $236102(2008)$.

[8] S.D. Findlay, M.P.Oxley, S. J. Pennycook and L.J. Allen, Ultramicroscopy 104, 126 (2005)

[9] P. Lu et al, Nano Lett. To be submitted (2013).

[10] Sandia National Laboratories is a multi-program laboratory managed and operated by Sandia Corporation, a wholly owned subsidiary of Lockheed Martin Corporation, for the US Department of Energy's National Nuclear Security Administration under contract DE-AC04-94AL85000. The work at Los Alamos was performed, in part, at the Center for Integrated Nanotechnologies, an Office of Science User Facility operated for the U.S. Department of Energy (DOE) Office of Science. Los Alamos National Laboratory, an affirmative action equal opportunity employer, is operated by Los Alamos National Security, LLC, for the National Nuclear Security Administration of the U.S. Department of Energy under contract DE-AC52-06NA25396.

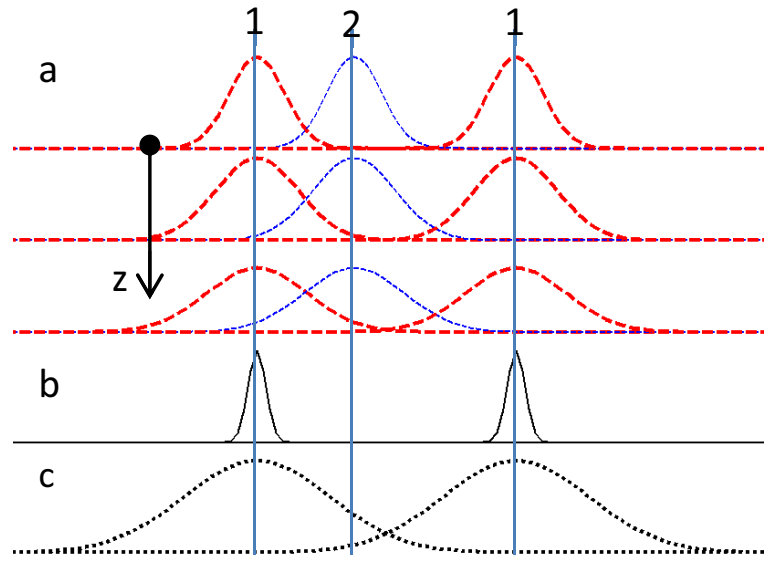

Fig.1. Schematic showing the x-ray generation process in STEM-EDS: (a) Gaussian electron probes at different thickness $(\mathrm{z})$ illustrating beam broadening along $\mathrm{z}$. Position 1 is at the atomic column site and position 2 is away from the site; (b) effective EDS scattering potential localized at the atomic column and (c) overall x-ray profile obtained by the convolution of electron wave at different thickness with EDS scattering potential and integration of the contribution through the sample thickness.
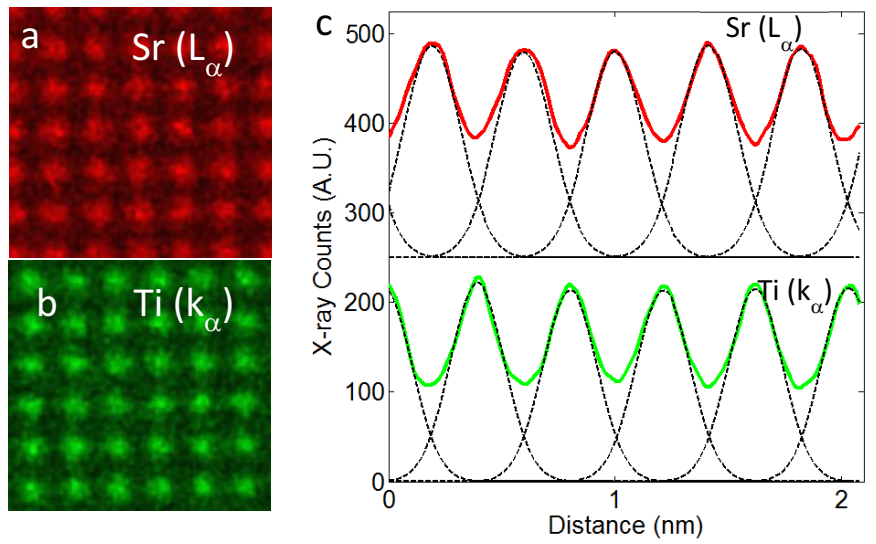

Fig.2: (a) $\mathrm{Sr} \mathrm{L}_{\alpha}$ and (b) Ti $\mathrm{K}_{\alpha}$ EDS maps extracted from STEM-EDS spectral imaging dataset of a thin $\mathrm{SrTiO}_{3}$ sample in [100] direction; (c) the x-ray profiles along the horizontal axis, along with the Gaussian peaks fitted to the profiles. A FEI Titan G2 80-200 STEM with Cs probe corrector and superX-EDS detector (electron probe with size of less than $0.18 \mathrm{~nm}$, and convergence angle of 18.0 mrad and probe current of $\sim 100 \mathrm{pA}$ ) was used for data collection. 